\title{
Validating the Simulation of Large-Scale Parallel Applications Using Statistical Characteristics
}

\author{
DELI ZHANG, University of Central Florida \\ JEREMIAH WILKE and GILBERT HENDRY, Sandia National Laboratories \\ DAMIAN DECHEV, University of Central Florida, Sandia National Laboratories
}

\begin{abstract}
Simulation is a widely adopted method to analyze and predict the performance of large-scale parallel applications. Validating the hardware model is highly important for complex simulations with a large number of parameters. Common practice involves calculating the percent error between the projected and the real execution time of a benchmark program. However, in a high-dimensional parameter space, this coarse-grained approach often suffers from parameter insensitivity, which may not be known $a$ priori. Moreover, the traditional approach cannot be applied to the validation of software models, such as application skeletons used in online simulations. In this work, we present a methodology and a toolset for validating both hardware and software models by quantitatively comparing fine-grained statistical characteristics obtained from execution traces. Although statistical information has been used in tasks like performance optimization, this is the first attempt to apply it to simulation validation. Our experimental results show that the proposed evaluation approach offers significant improvement in fidelity when compared to evaluation using total execution time, and the proposed metrics serve as reliable criteria that progress toward automating the simulation tuning process.
\end{abstract}

Categories and Subject Descriptors: C.4.3 [Performance of Systems]: Measurement Techniques

General Terms: Measurement, Experimentation

Additional Key Words and Phrases: Simulation evaluation, evaluation metrics, software skeleton

ACM Reference Format:

Deli Zhang, Jeremiah Wilke, Gilbert Hendry, and Damian Dechev. 2016. Validating the simulation of largescale parallel applications using statistical characteristics. ACM Trans. Model. Perform. Eval. Comput. Syst. 1, 1, Article 3 (February 2016), 22 pages.

DOI: http://dx.doi.org/10.1145/2809778

\section{INTRODUCTION}

Building a system simulator is a well-known approach for predicting the performance of large-scale parallel applications. ${ }^{1}$ Performance projections can be produced by executing the application in a simulated environment comprised of hardware models in place

\footnotetext{
${ }^{1}$ Since Message Passing Interface (MPI) is the dominating parallel programming model in high performance computing, we use the term large-scale parallel application and MPI application interchangeably.
}

Sandia National Laboratories is a multiprogram laboratory managed and operated by Sandia Corporation, a wholly owned subsidiary of Lockheed Martin Corporation, for the U.S. Department of Energy's National Nuclear Security Administration under contract DE-AC04-94AL85000.

Authors' addresses: D. Zhang and D. Dechev, Department of Computer Science, University of Central Florida, 211 Harris Center (Building 116), 4000 Central Florida Boulevard, Orlando, FL 32816 USA; emails: de-li.zhang@knights.ucf.edu, dechev@eecs.ucf.edu; J. Wilke and G. Hendry, Sandia National Laboratories, California P.O. Box 969, Livermore, CA 94551-0969; emails: \{jjwilke, ghendry\}@sandia.gov.

Permission to make digital or hard copies of part or all of this work for personal or classroom use is granted without fee provided that copies are not made or distributed for profit or commercial advantage and that copies show this notice on the first page or initial screen of a display along with the full citation. Copyrights for components of this work owned by others than ACM must be honored. Abstracting with credit is permitted. To copy otherwise, to republish, to post on servers, to redistribute to lists, or to use any component of this work in other works requires prior specific permission and/or a fee. Permissions may be requested from Publications Dept., ACM, Inc., 2 Penn Plaza, Suite 701, New York, NY 10121-0701 USA, fax +1 (212) 869-0481, or permissions@acm.org.

(c) 2016 ACM $2376-3647 / 2016 / 02-A R T 3 \$ 15.00$

DOI: http://dx.doi.org/10.1145/2809778 
of hardware components, which is a process that can vary depending on the structure of the simulator and the granularity of the models used. To fully capture the interactions between computation and communication in hardware and software components, system simulators usually evolve into large software packages comprised of several layers of modules with dozens of input parameters. Regardless, using coarse-grained models for simulations of large parallel applications at scale is an efficient approach in exploring the complex design space of supercomputer hardware and software.

Such complexity makes the task of establishing a simulator's accuracy a challenging problem. Evaluating the accuracy of simulation, or simulation validation, normally consists of three core components: an evaluation metric, an error measure, ${ }^{2}$ and benchmark programs. Most hardware simulation frameworks evaluate accuracy in terms of total execution time [Hoefler et al. 2010; Hammond et al. 2009; Denzel et al. 2010; Susukita et al. 2008; Zhai et al. 2010], where the projected execution time is compared against the real execution time and the error of the simulation is denoted by a percentage ratio. While the total execution time is one of the most important artifacts in a simulation environment, it lacks the fidelity (the ability to identify execution details) and the coverage (the ability to encompass all aspects of execution) to serve as a metric for fine-grained simulation accuracy.

Validation also applies to software models, such as skeleton applications [Sottile et al. 2013], which are used in online simulators as the surrogates of the original program. A skeleton is derived by removing computationally intensive program fragments, but retaining code that determines the execution behavior. The program's communication pattern has the most significant impact on the performance and scalability of largescale applications. Validating a skeleton is similar to machine model validation except that now we compare the execution of two different programs (the original program and its skeleton) on the same simulated environment.

In this article, we present a validation approach that aims to capture fine-grained execution details. ${ }^{3}$ It consists of evaluation metrics that decompose execution time and communication traffic into finer granularity, and an unbiased error measure that quantifies the disparity between corresponding metrics of two executions. The proposed metrics take into account five groups of statistical data profiled from program traces: overall traffic and timing, per-node traffic and timing, MPI function histogram, collective synchronization, and node-to-node communication. Similar metrics have been used in the task of performance optimization [Vetter and McCracken 2001; Kamil et al. 2010], yet this is the first attempt to apply them to simulation validation through quantitative comparison. On the other hand, a trace file such as one collected with the DUMPI format [Janssen et al. 2010], can provide thorough details about the execution, but quantitatively comparing two traces is difficult because the relevant information is scattered among a large volume of data. Our statistical metrics aggregate the relevant information from the traces and provide a scalable and lightweight overview of the application's execution details.

Furthermore, we outline the design and use of a validation toolset, based on our accuracy metrics and error function, for the analysis, comparison, and visualization of traces. To verify our approach, we conduct a number of trace-driven hardware model validation experiments on the Structural Simulation Toolkit/macro simulation framework [Janssen et al. 2010] with two representative scientific applications. The major contributions of this work include:

\footnotetext{
${ }^{2}$ We use the term error measure to refer to the function that calculates the error between two values.

${ }^{3}$ This work extends the authors' previous conference publication [Zhang et al. 2014]. We (1) introduce the metrics for network traffic; (2) review literature on simulation validation and error measurements; and (3) explain in detail the results of the hardware model validation experiments.
} 
(a) We present the first validation methodology using fine-grained accuracy metrics. Our approach aims to address the parameter insensitivity problem.

(b) According to our experimental evaluation, our approach reveals a higher degree of details when compared with validation using total execution time.

(c) We also demonstrate that the proposed metrics serve as reliable criteria for identifying the optimal hardware model.

(d) Our toolset has been successfully applied to verify the software skeletons produced by the autoskeletonization process [Sottile et al. 2013].

The rest of the article is organized as follows. Section 2 covers the background on the SST/macro simulation framework and the parameter insensitivity problem. Section 3 details the proposed statistical metrics and design of the toolset. In Section 4, we present our experimental evaluation. Section 5 summarizes the related works. The final section concludes with perspectives on future work.

\section{SIMULATION AND PARAMETER INSENSITIVITY}

The simulation of MPI applications has been an important research problem and many new frameworks are still being proposed today [Clauss et al. 2011; Desprez et al. 2011; Núñez et al. 2010; Zhai et al. 2010]. Existing simulation frameworks can be divided into two categories: online simulation, also called simulation via direct execution where the original or slightly modified program is executed within the simulation environment, and off-line or trace-driven simulation where the trace of a program running on the real machine is "replayed" through the simulator. SST/macro [Janssen et al. 2010] is a coarse-grained system simulator that supports both online and off-line simulations. When it is driven by a trace file in off-line mode, the simulator accepts trace files in both the standard Open Trace Format (OTF) and its custom DUMPI format. When operating in on-line mode, the simulator can execute either an unmodified MPI application or a skeleton application derived from the original application [Sottile et al. 2013]. The use of skeletons allows the accelerated simulation of the essential communication pattern without the burden of heavy computation.

SST/macro is a key component in the hardware/software codesign process, one that addresses the difficult problem of designing future exascale parallel computers with scalable software performance, manageable power consumption, and reasonable cost [Shalf et al. 2011]. The scale and complexity of supercomputers have increased exponentially over the last decade and are expected to grow continuously. However, porting and tuning existing code to these machines is an increasingly difficult task as parallelism takes over for traditional Moore's Law performance scaling. Furthermore, hardware architects have to account for power and cost constraints and deal with extreme parallelism on an even higher level when designing future exascale systems. Hardware and software codesign is an iterative process to optimize both the application and the hardware for some combination of performance, power, and cost. Simulation plays a key role in this process because it enables quantitative exploration of system design trade-offs before the system becomes available [Janssen et al. 2011]. Multiple levels of simulation are needed in this process, from the most crude constitutive models to cycle accurate simulation [Rodrigues et al. 2011; Underwood et al. 2007]. SST/macro is positioned between these two extremes, thus it trades some simulation accuracy for greater scalability.

For existing simulation approaches, validation using total execution time is vulnerable to the parameter insensitivity problem, which occurs in regions of high-dimensional parameter space. Consider a typical system simulator that models a target machine with a set of hardware parameters (e.g., network bandwidth, memory latency, and network topology). Each parameter characterizes a different aspect of the simulated 
hardware components. The underlying simulation model determines the way in which these parameters affect the outcome of a simulation. However, given two or more slightly different parameter sets, it is very likely they project similar timings. Possible reasons for this phenomenon are (1) the changed parameter makes trivial contribution to the total execution time (low parameter sensitivity in the region of the parameter space considered); and (2) several changed parameters have adversarial effects that cancel each other (e.g., increasing bandwidth and latency at the same time). Identifying the most accurate hardware model is not possible without using complimentary criteria. We argue that even when the execution time is identical, the underlying structure of the executions can be different, which may not be manifested until the models are stressed beyond the validated condition.

\section{VALIDATION METHODOLOGY}

Our core validation process consists of two steps: trace analysis and trace comparison. The process takes as input two traces and quantifies the disparity between them. In the case of hardware model validation, these two traces come from executing the benchmark on the real machine and the simulator. In the case of skeleton validation, they are from the skeleton and the original application executed on the simulator using the hardware model. The first step extracts five groups of statistics from each trace, which are used as the fine-grained evaluation metrics. The second step computes the element-wise percentage difference on a per-group basis between the extracted metrics. In addition to the error values, which give a quick overview of the validation result, we also keep the intermediate statistical data for a detailed exploration of the differences.

\subsection{Trace Analysis}

Trace analysis aggregates timing and traffic information from function call logs in a trace file. The core idea is to decompose traffic and timing information along different dimensions to obtain various views of the same data. At the topmost level, we simply separate the total computation and communication time. As we progress, the granularity of the decomposition increases. Namely, we break down the traffic and timing on a per-node, per-function, per-collective-phase, and node-to-node basis. These metrics cover both internode and intranode execution characteristics and they also strike a balance between granularity and noise. While trace files provide fine-grained execution details, isolated function logs are noisy. By aggregating the information, we diminish the noise and distill statistically significant characteristics.

For the rest of the article, we represent the number of nodes by $N$, the number of MPI functions by $F$, the number of collective phases by $S$, and the total occurrence of a function by $M$. We also use subscripts $i, j, k, l$ to identify the node, function, occurrence, and collective phase, respectively. For example, the block time of the function $j$ on node $i$ occurring for the $k$ th time is given by $T_{i, j, k}$.

The first metric is the overall traffic and timing, which lists the respective total of communication traffic $(T R)$, block time $(B T)$, and compute time $(C T)$ given by

$$
\begin{gathered}
T R=\sum_{i<N} \sum_{j<F} \sum_{k<M} B_{i, j, k}, \\
B T=\sum_{i<N} \sum_{j<F} \sum_{k<M} T_{i, j, k}, \\
C T=T O T A L-B T,
\end{gathered}
$$

where $B_{i, j, k}$ is the traffic of a single MPI function call on one node and $T_{i, j, k}$ represents the block time in the same way. The traffic measures the communication dataflow, 
counting the total number of data bytes sent out by each node. It is a necessary measurement for skeleton validation, in which the preservation of communication behavior is verified by the error of the traffic. Note that the traffic is not necessarily measuring the exact bytes of data going through the network, which varies depending on the MPI implementation, but rather the total data volume passed through send/recv buffers. Block time is the amount of time spent on blocking communication calls. Comparing with total execution time, listing both block time and compute time breaks down timing granularity, allowing us to capture the communication and computation performance separately.

The second metric, per-node traffic and timing, decomposes the traffic and timing by node. The timings and traffic for each node form a $3 \times N$ matrix:

$$
\left|\begin{array}{lll}
T R_{0} & \cdots & T R_{N} \\
B T_{0} & \cdots & B T_{N} \\
C T_{0} & \cdots & C T_{N}
\end{array}\right|,
$$

where $(B T)$ and $(C T)$ denote block time and compute time, respectively. We also collect per-node traffic $(T R)$. These breakdown statistics reflect the load balancing among the nodes, because we expect an accurate simulation to also reproduce the deviation among the nodes.

$$
\begin{aligned}
T R_{i} & =\sum_{j<F} \sum_{k<M} B_{i, j, k}, \\
B T_{i} & =\sum_{j<F} \sum_{k<M} T_{i, j, k}, \\
C T_{i} & =\text { TOTAL } L_{i}-B T_{i} .
\end{aligned}
$$

The third metric is the MPI function histogram. For each profiled MPI function call, we record the total traffic $(T R)$ and block time $(B T)$ on each node. They form two $F \times N$ matrices:

$$
\begin{aligned}
& \left|\begin{array}{ccc}
T R_{00} & \cdots & T R_{0 N} \\
\vdots & \ddots & \vdots \\
T R_{F 0} & \cdots & T R_{F N}
\end{array}\right|, \\
& \left|\begin{array}{ccc}
B T_{00} & \cdots & B T_{0 N} \\
\vdots & \ddots & \vdots \\
B T_{F 0} & \cdots & B T_{F N}
\end{array}\right|,
\end{aligned}
$$

where

$$
\begin{aligned}
& T R_{i, j}=\sum_{k<M} B_{i, j, k}, \\
& B T_{i, j}=\sum_{k<M} T_{i, j, k} .
\end{aligned}
$$

We also collect the call counts $(C C)$ for each MPI function across all nodes.

$$
C C_{j}=\sum_{i<N} \sum_{k<M} 1
$$


Similar to the traffic information in previous metrics, this measurement of communication behavior is essential for the verification of program skeletons because the skeletonization process may accidentally slice out MPI functions [Sottile et al. 2013].

The fourth metric is the collective synchronization. We define a collective phase as an interval of program execution between two consecutive collective MPI calls. Unlike point-to-point functions, collective functions operate on every node within a communicator, and they effectively act as the barriers that synchronize program execution across all nodes. The sequence of collective function calls between MPI_Init and MPI_Finalize divide the whole execution into numbers of synchronization phases. The time interval of these phases on all nodes also forms a $S \times N$ matrix:

$$
\left|\begin{array}{ccc}
S T_{00} & \cdots & S T_{0 N} \\
\vdots & \ddots & \vdots \\
S T_{S 0} & \cdots & S T_{S N}
\end{array}\right|
$$

where $S T_{i, l}$ denotes the duration of a single collective phase $l$ on node $i$. We then compute the average $(A S)$ and the standard deviation $(S S)$ as follows:

$$
\begin{gathered}
A S_{l}=\frac{1}{N} \sum_{i<N} S T_{i, l}, \\
S S_{l}=\sqrt{\frac{1}{N} \sum_{i<N}\left(S T_{i, l}-A S_{l}\right)^{2} .}
\end{gathered}
$$

The last metric is the node-to-node communication, which reveals the communication traffic and timing among all pairs of nodes. Node-to-node traffic forms a $N \times N$ matrix:

$$
\left|\begin{array}{ccc}
T R_{00} & \cdots & T R_{0 N} \\
\vdots & \ddots & \vdots \\
T R_{N 0} & \cdots & T R_{N N}
\end{array}\right|
$$

where $T R_{i i^{\prime}}$ denotes the total amount of data transmitted from node $i$ to node $i^{\prime}$. The traffic sparse matrix has been widely used to study the communication patterns. Here we define a node-to-node timing matrix in the same spirit:

$$
\left|\begin{array}{ccc}
B T_{00} & \cdots & B T_{0 N} \\
\vdots & \ddots & \vdots \\
B T_{N 0} & \cdots & B T_{N N}
\end{array}\right|
$$

where $B T_{i i^{\prime}}$ denotes the total blocking communication time between two nodes $i, i^{\prime}$.

\subsection{Trace Comparison}

The timing matrices (Equations (4), (9), (13), and (17)) contain fine-grained decomposition of total execution time; we use them in our validation experiments to evaluate the fitness of hardware models. In a typical validation setting, we obtain four pairs of timing matrices from two input traces after the trace analysis process. For each matrix pair, we first compute the element-wise percentage difference and then take the average as final error:

$$
E r r=\frac{1}{n} \sum_{1 \leq i \leq n} \frac{\left|x_{i}-r_{i}\right|}{\frac{x_{i}+r_{i}}{2}}
$$


Unlike percentage error, percentage difference only measures magnitude and is completely symmetric. This can be observed from $|x-r|=|r-x|$. A symmetric error function is important when comparing two simulated executions, because we have no prior knowledge to prefer one over the other, and we expect the same error regardless which one is used as a reference. Other established methods that compute disparities among matrix data, for example, root-mean-square error, correlation, and Frobenius norms, produce roughly identical results as the element-wise percentage difference.

In a skeleton generation process, the traffic data (Equations (4), (8), (12), and (16)) are used to validate the software skeletons: the quantitative differences are feedback to user to iteratively improve the quality of the skeletons. During this iterative process, the intermediate skeleton programs may not reproduce the exact behavior of the original program, which may be overcome by manual analysis of the execution difference. The difference between a skeleton and its parent may also be introduced deliberately, such as different choices of communication functions. For example, one might replace the general MPI_Send with MPI_Ssend to study the effect of blocking synchronization. Under these circumstances, we also compute an additional quantitative error using traffic data that highlights the structural difference between the statistics metrics. We present this structural error alongside the regular quantitative error in our comparison report.

\subsection{Toolset Design}

We put our proposed statistical approach to practice by creating a simulation validation toolset. The validation toolset consists of three loosely coupled components: a Trace Analysis Tool (TAT), a Trace Comparison Tool (TCT), and an additional Trace Visualization Tool (TVT) that helps render the analysis and comparison results in graphs. The tools, which are implemented in $\mathrm{C}++$, are coupled together by a common XML format, facilitating both stand-alone invocation and scripted automation. TAT transforms the trace files into statistics report files in XML format. Each statistics group is a modular entry in the report file. The advantage of this structured file format is that new entries can be added without interfering with the existing ones. This allows us to tailor the analysis process according to the simulation framework used.

The DUMPI tracing library [Janssen et al. 2010] produces trace files that drive SST/macro in off-line mode. We prioritize the support of DUMPI format in our toolset because of the following performance advantages over Open Trace Format. First, DUMPI differentiates between variations of MPI calls (e.g., MPI_Send, MPI_Ssend, MPI_Bsend). Second, it records full input and output argument listings other than simple payload data. For example, vector arguments for MPI collectives and detailed tracking for MPI request calls are all captured. Finally, DUMPI minimizes collection overhead and trace file size to reduce impact on profiled timings. For each type of value stored in the binary file, DUMPI chooses the minimal bit width that suits the range of the value. For example, there are over 300 MPI functions, so it employs 16-bit integers to identify them.

\section{VALIDATION EXPERIMENT}

In this section, we validate the accuracy of SST/macro simulations against Hopper with two prevalent High-Performance Computing (HPC) mini-applications: MiniMD and CoMD. We focus on hardware model validation in the following sections. The goal of our experiments is twofold: (1) based on the fine-grained timing decompositions, to reveal possible causes of the disparity between the simulation and the actual execution; and (2) based on the quantized errors, to evaluate the fidelity of the proposed metrics in identifying the optimal hardware model. By optimal we mean that the hardware 
Table I. Nominal Parameters Modeling Hopper

\begin{tabular}{l|c|c}
\hline Parameters & Nominal Value & Nominal Value \\
\hline BTE injection bandwidth and latency & $7 \mathrm{~GB} / \mathrm{s}$ & $0.5 \mu \mathrm{s}$ \\
FMA injection bandwidth and latency & $8 \mathrm{~GB} / \mathrm{s}$ & $0.2 \mu \mathrm{s}$ \\
Link bandwidth and latency & $2.2 \mathrm{~GB} / \mathrm{s}$ & $70 \mathrm{~ns}$ \\
Switch bandwidth and latency & $1.8 \mathrm{~GB} / \mathrm{s}$ & $30 \mathrm{~ns}$ \\
Node memory bandwidth and latency & $10.4 \mathrm{~GB} / \mathrm{s}$ & $6 \mathrm{~ns}$ \\
\hline
\end{tabular}

model produces a simulation result that has minimal error when compared against the reference execution on the target machine.

Hopper is a Cray XE6 cluster with a Gemini interconnect. ${ }^{4}$ Hopper nodes employ a Nonuniform Memory Access (NUMA) architecture with four AMD Opteron 6100 processors (six cores per chip). The Network Interface Controller (NIC) is connected to the L3 cache of one processor via HyperTransport. The Gemini NIC provides two separate paths. The Fast Memory Access (FMA) path is optimized for low-latency 8-64 byte puts/gets. FMA is intended for small messages as it still requires source-side synchronization. The second path—the Block Transfer Engine (BTE)—has a higher latency, but provides full asynchronous RDMA and is optimized for transferring large blocks directly to memory. A 48-port Yarc switch connects two NICs in the three-dimensional (3D) torus network. Each router has 10 links, four each in $x$ and $z$ dimension and two in the $y$ dimension. Each link provides $2.2 \mathrm{~GB} / \mathrm{s}$ observable bandwidth. Table I lists the nominal parameters derived by executing small ping-pong benchmarks with customized lightweight MPI implementations on Hopper. Some of the nominal values differ considerably from the values quoted by Cray specification, because our MPI implementation simplifies the MPI protocol and turns off adaptive routing to facilitate machine model validation. Also, we list bandwidth as observed values that exclude packet overhead.

MiniMD and CoMD are two proxies from the Mantevo package ${ }^{5}$ for the force computation in a typical molecular dynamics application. These mini-applications serve as appropriate benchmarks for all around testing of the simulator because they utilize both point-to-point and collective communication. We first obtain reference DUMPI traces by running the two benchmarks with strong scaling: for miniMD we do this on $64,128,256$, and 512 nodes without intranode allocation; for CoMD we execute it on $32,64,128$, and 256 nodes with intranode allocation, which is equivalent to $768,1,536$, 3,072 , and 6,144 MPI processes. We then collect simulation traces by replaying the reference traces on SST/macro with the nominal hardware model. When replaying traces, SST/macro simulates the target machine's network traffic deterministically using a hybrid packet and flow model. Any disparity between the simulation execution and the real execution is an artifact of the imperfect model. Obtaining confidence intervals of the simulation through multiple executions of the same simulation configuration is not necessary, as the simulation error given by both coarse-grained and fine-grained metrics is a direct measure of the quality of the hardware model.

\subsection{Fine-Grained Differences}

In this section, we manually analyze the simulation traces of miniMD using the trace analysis tool to illustrate the fine-grained execution differences. We use the term "nominal model" to refer to the set of parameters that were derived from machine specifications. Given the way in which the various hardware components are modeled and other factors, this is usually not the most accurate predictor of performance. In Table II,

\footnotetext{
${ }^{4} \mathrm{http} / / /$ www.cray.com/Products/Computing/XE/XE6.aspx.

${ }^{5} \mathrm{http}: / /$ www.mantevo.org/packages.php.
} 
Table II. Percentage Error for miniMD

\begin{tabular}{l|c|c|c|c}
\hline Nodes & 64 & 128 & 256 & 512 \\
\hline Error & $-0.42 \%$ & $-1.67 \%$ & $-5.24 \%$ & $-29.1 \%$ \\
\hline
\end{tabular}

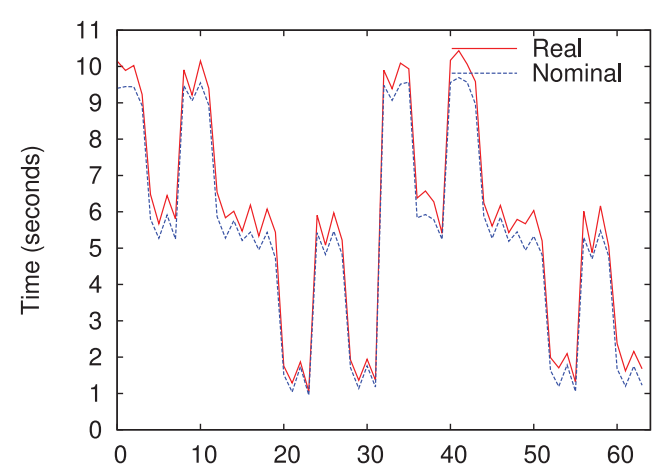

(a) 64 nodes

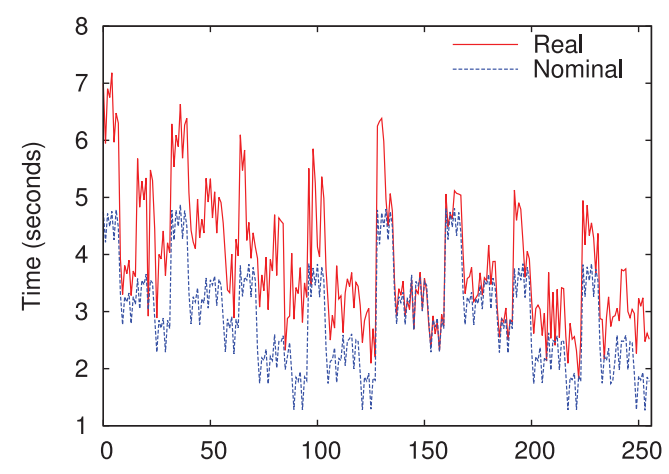

(c) 256 nodes

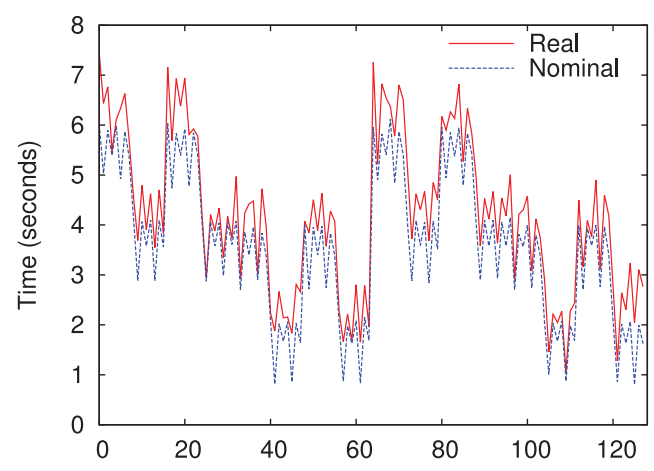

(b) 128 nodes

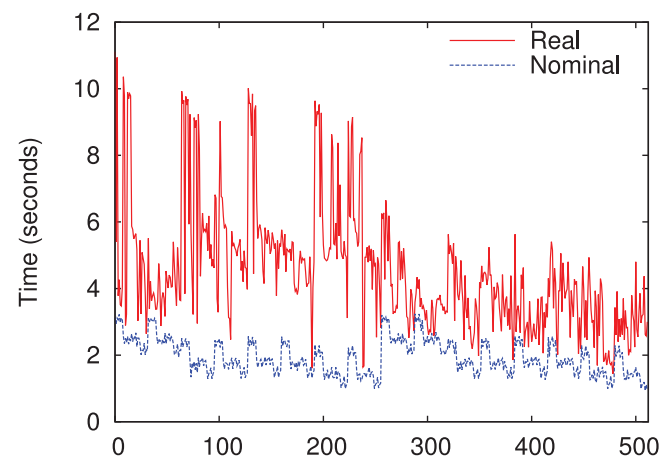

(d) 512 nodes

Fig. 1. Per-node timings for miniMD.

we show the percentage error of projected total execution time against real execution time. Here we follow the convention of using percentage error to denote the error of execution time, but in the rest of the article we use percentage difference for all error measurements. All simulation results agree with the real execution within $-5 \%$ error except for the case of 512 nodes, where the error jumps to $-29 \%$. Besides these facts, total execution time does not reveal any insight into the reasons for the increasing error margins.

In Figure 1, we show the per-node breakdown of communication timings. The $x$-axis denotes the number of nodes, and the $y$-axis denotes the execution time on each node. For 64 nodes, we observe a close fit of simulation timings on the node level, but there are some noticeable differences between the simulation and real execution on 128 nodes. As the number of nodes increases, the real timings become more noisy and deviate from the projections by a large margin. For example, on 256 nodes the error given the pernode timings metric is $13 \%$, while the error magnitude given by total execution time (Table II) is $5.24 \%$. The magnitude of the error does not necessarily reflect the fidelity of a metric, but a fine-grained metric usually produces a larger percentage difference than a coarse-grained metric due to its sensitivity to parameter changes. Per-node 


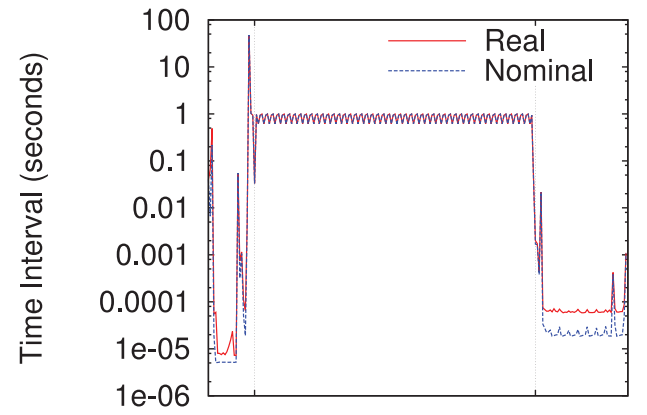

(a) 64 nodes

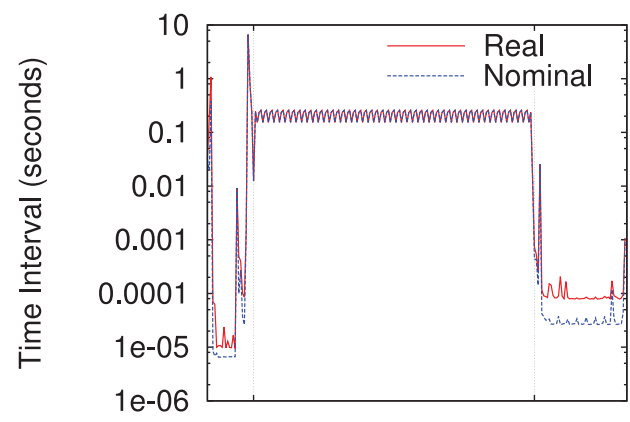

(c) 256 nodes

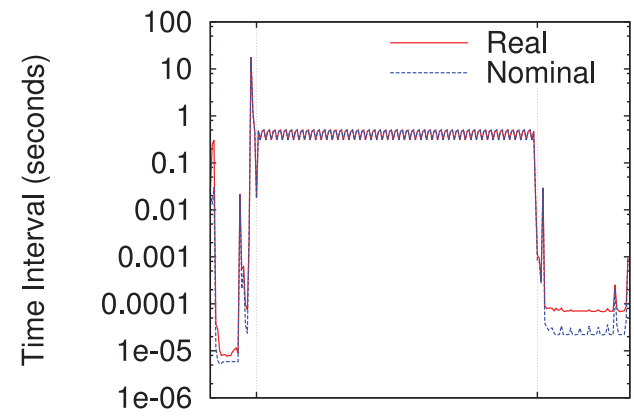

(b) 128 nodes

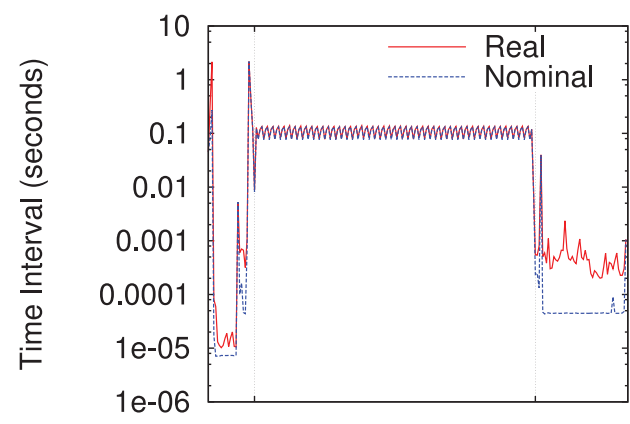

(d) 512 nodes

Fig. 2. Collective synchronization of 227 collective phases for miniMD.

decomposition of timings captures the mismatch of communication load among nodes, thus providing fidelity improvement over the total execution time.

Figure 2 displays the average timings of 227 collective phases for miniMD for all scales. The $x$-axis represents the collective phase, and the $y$-axis denotes the execution time of each phase. The executions can be divided into three consecutive stages as marked by two vertical dashed lines in the graphs. In the first stage, miniMD calls MPI_Bcast to setup computation. The sudden spike of the real execution is not entirely captured by the simulation because of the delay of broadcast function on the real machine (Figure 3). The second stage is dominated by heavy computation. We see that the execution time of the nominal model agrees with the real execution time. This is because in off-line simulation, the computation time is reproduced as the exact values captured by the traces (i.e., generally the part not being modeled). The last stage consists mainly of collective calls. In this stage, the projected execution time given by nominal model diverges from the time of real executions. For example, the projected execution time is $60 \%$ less than the real execution time on 128 nodes. Similar to the setup stage, the last stage consists mainly of collective calls, so it is vulnerable to system noise.

In Figure 3, we show the timing breakdown among MPI functions. MiniMD makes use of 11 MPI functions, ignoring the use of MPI_Comm_size and MPI_Comm_rank, which typically do not block the user process (and therefore do not get traced by DUMPI). In Figure 3, each bar denotes the average communication time by stacking nine MPI 


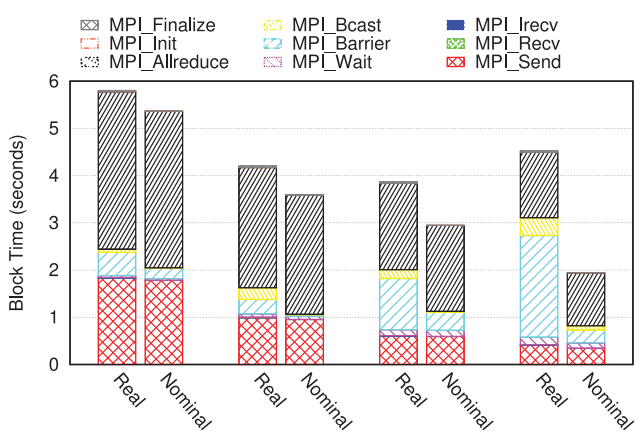

Fig. 3. MPI function histogram for miniMD.

functions together and the bars are clustered by number of nodes. It illustrates the structural difference of the composition of communication time. Note the time of each function is the sum of its blocking time across all nodes as described in Equation (11). MPI_Send is used by miniMD as point-to-point communication among the immediate neighbors. The nominal model gives accurate simulation of MPI_Send and MPI_Allreduce, with less than $4 \%$ error up to 256 nodes. The disparity between the real runs and the simulations contributed by MPI_Barrier is mostly due to resource contention from the jobs executed by other users. During the actual execution on Hopper, the allocated nodes are not isolated from other jobs. Background interference from them contributes to the systematical delay of the real run. As nodes grow, the delay in global communication causes slowdown on all nodes. Especially in the case of 512 nodes, MPI_Barrier makes up $40 \%$ of total communication time.

Given the knowledge of detailed job configurations, a simulation framework such as $\mathrm{SST} / \mathrm{macro}$ is able to model contention from other jobs. However, the detailed information of the other jobs on Hopper is inaccessible to us during our experiment, thus we treated performance fluctuation caused by such contention as background noise. On the other hand, even without detailed knowledge of contending jobs, a suitable mapping of processes to the nodes can substantially improve communication performance by reducing network congestion [Peña et al. 2013; Sudheer and Srinivasan 2012; Albing et al. 2011]. We could thus alleviate the impact of contending jobs in real world by allocating adjacent nodes for the target job. The communication among a group of closely located nodes is less likely to be interfered by contending jobs because the communication paths are short and local. This can also be observed in Figure 1, where the per-node communication time pattern on Hopper is more accurately captured by the simulation results with less number of nodes.

In Figure 4, we show the absolute difference between the simulated and real timing matrices mapped to color space. Plots are sparse matrices in which the color of each pixel represents the error intensity at its coordinate. The $x$ - and $y$-axes of each subplot denote the node rank. On all scales, we observe that the error in block time of the collective functions propagates through all nodes, forming the horizontal lines. The error in local point-to-point communications forms a dotted pattern along the diagonal. The color tone for 512 nodes differs considerably from the other scenarios, supporting the significantly larger error given by total execution time.

Overall, the analysis of the four fine-grained metrics shows that (1) they support each other by revealing the influences of global background noise on the real machine; and (2) they cover different characteristics that form a whole picture of the execution when combined together. 

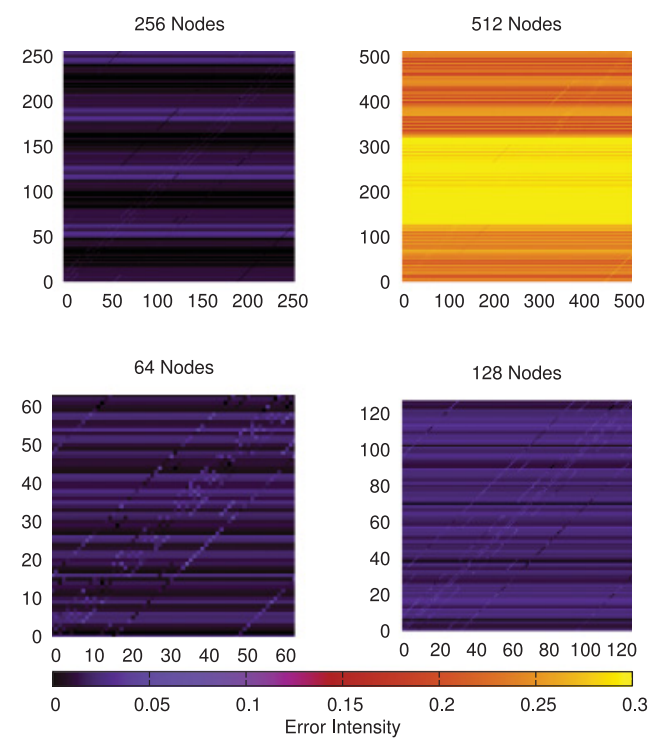

Fig. 4. Node-to-node time error for miniMD.

Table III. Percentage Error for coMD

\begin{tabular}{l|c|c|c|c}
\hline Processes & $768(32$ Nodes $)$ & $1,536(64$ Nodes $)$ & $3,072(128$ Nodes $)$ & 6,144 (256 Nodes) \\
\hline Error & $-0.324 \%$ & $-2.84 \%$ & $-4.28 \%$ & $-16.1 \%$ \\
\hline
\end{tabular}

\subsection{Toward Automatic Simulation Validation}

We also performed an automated parameter sweep experiment using coMD to verify the improved fidelity of our fine-grained metrics over total execution time. This brings us a step closer to an automatic simulation tuning framework that calibrates the simulator (i.e., finding the optimal simulation parameters) with little or no human intervention. In each sweep experiment, we alter one pair of the parameters in Table I while keeping other parameters in their nominal value. For each parameter, we sample 20 values in a geometric series, so the whole parameter space of one experiment consists of 400 (20 by 20 ) value pairs. Finally, we obtain quantized error for each simulation by comparing the traces from the simulations against the reference traces using our trace comparison method. Ideally, we expect the simulation with nominal parameter values to produce execution trace that has minimal error when compared against the reference trace. Future autotuning process would heuristically search the whole parameter space and favor those that produce the most similar traces. In Table III, we show the percentage error of coMD's projected execution time against real execution time using nominal parameters.

In Figure 5(a), we show the errors of projected execution time compared to real execution time when sweeping network link bandwidth and latency on 768 processes. The link bandwidth is modeled as link frequency by the simulator, so the nominal value can be obtained as follows: $2.2 \mathrm{~GB} / \mathrm{s}=2.4 \mathrm{GHz} \times 1.5 \mathrm{bytes} / \mathrm{cycle} \times 0.6$ where 1.5 bytes/cycle denotes the 12 channels of the Gemini torus links (i.e., 12 bits), and the payload fraction 0.6 means only $60 \%$ of the traffic is used as payload. By total execution time we mean the execution time of the program. To eliminate interferences from setup, we report the total execution time as the interval between MPI_Init and MPI_Finalize on the root process. Each point on the $x-y$ plane corresponds to a simulation configuration using associated parameters, and the $z$-axis represents the error of the simulation measured 

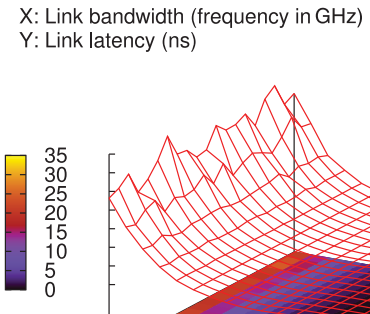

Error (\%)

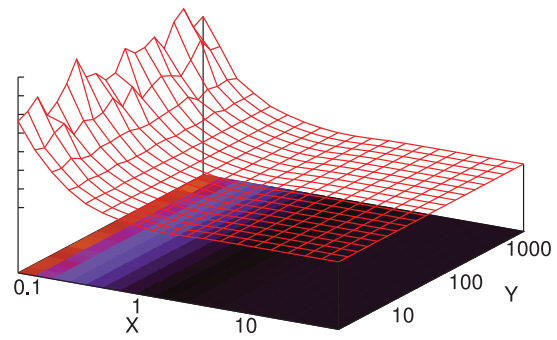

(a) Total execution time 768 processes

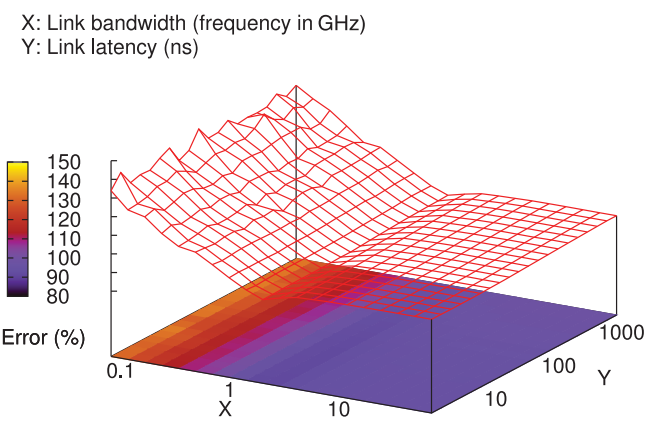

(c) MPI histogram 1,536 Processes

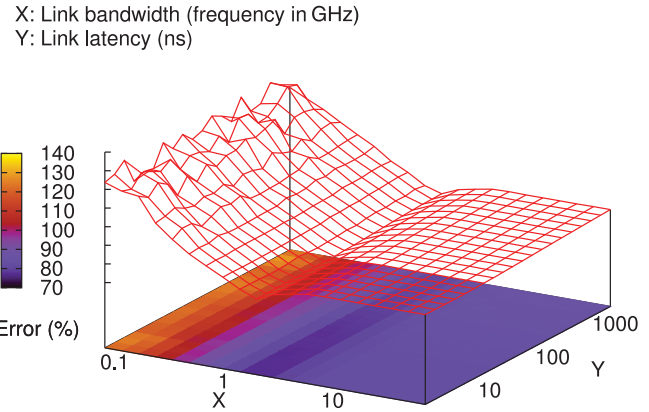

(b) MPI histogram 768 Processes

$\mathrm{X}$ : Link bandwidth (frequency in $\mathrm{GHz}$ )

Y: Link latency (ns)

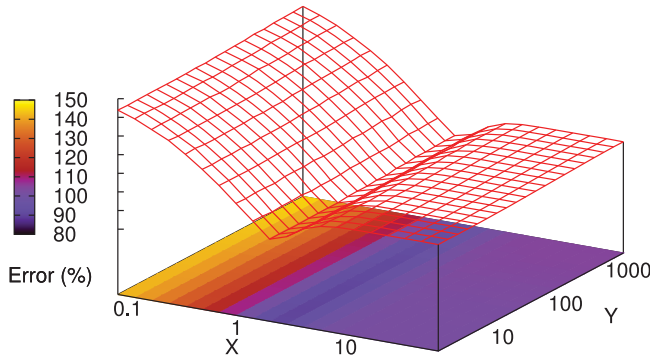

(d) MPI histogram 3,072 Processes

Fig. 5. Link bandwidth-latency sweeping for coMD.

by the total execution time. The error values are presented in percentage. We also map error value down to the $x-y$ plane using colored keys. We observe in Figure 5(a) that the errors given by the total execution time do not show a clear convergence trend: the errors almost keep constant in a flat area between $1 \mathrm{GHz}$ and $100 \mathrm{GHz}$. In Figure 5(b), we show the error value given by the MPI histogram metric. As marked by a dark strip in the figure, the error values given by this metric clearly converge at $2.2 \mathrm{~GB} / \mathrm{s}(2.4 \mathrm{GHz})$, which overlaps with the nominal value. The convergence also holds on larger scales, such as the scenario of 1,536 processes and 3,072 processes as illustrated in Figures 5(c) and 5(d). Node-to-node timing was also able to correctly capture the disparity caused by changing link bandwidth as illustrated in Figure 6(a). In addition, we also observe that in Figure 5(a), the errors keep constant along the $y$-axis. This is an instance of the parameter insensitivity problem, where changes in the network link frequency have little influence on the projected execution time. In Figure 6(b), we show the error given by the collective synchronization metric, which is sensitive to the link latency. The error reaches minimum at $2.2 \mathrm{~GB} / \mathrm{s}$ and $600 \mathrm{~ns}$ as marked by a dark area in the graph. Again the link bandwidth matches the nominal value, but the optimal latency is much larger than the nominal value of 70ns. Similar to the execution of miniMD (Figure 2), coMD is also computation bound for the major part of the execution. The simulation does not model noise, thus the simulation with larger latency produces a longer delay that matches the real execution, which would be preferred by the evaluation metric. 


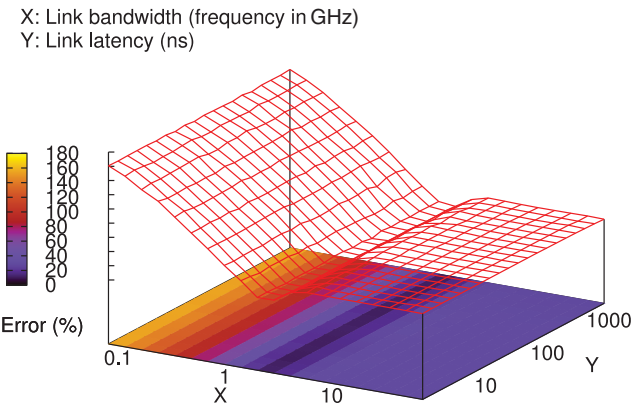

(a) Node-to-node timing 3,072 Processes
$\mathrm{X}$ : Link bandwidth (frequency in $\mathrm{GHz}$ )

Y: Link latency (ns)

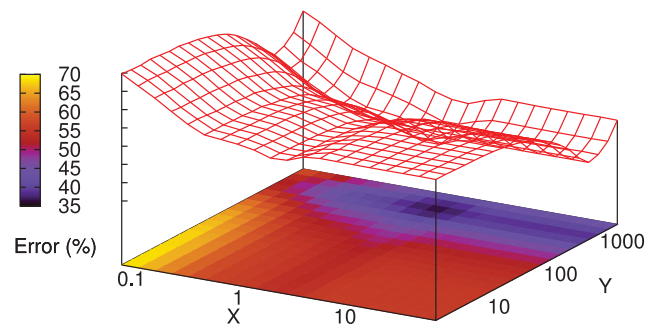

(b) Collective Synchronization 3,072 Processes

Fig. 6. Link bandwidth-latency sweeping for coMD.

In Figure 7, we show the error when sweeping the switch bandwidth and latency. The switch bandwidth can be obtained from switch frequency; for nominal value this is $1.8 \mathrm{~GB} / \mathrm{s}=1 \mathrm{GHz} \times 3$ bytes $/$ cycle $\times 0.6$ where 3 bytes/cycle denotes the 24-bit physical unit of Gemini router, and 0.6 is the payload fraction.

We observe in Figure 7(a) for 768 processes the errors of total execution time form a plateau and the minimal error is obtained when at extreme small bandwidth and extreme large latency. On larger scales, total execution time gives similar error graphs. Like the communication time of miniMD shown in Figure 3, the projected execution time of coMD is generally smaller than real execution time because of the presence of background noise. Smaller bandwidth and larger latency would erroneously prolong the projected execution time at the expense of extorting fine-grained execution details. For example, the extortion is captured by the collective synchronization metrics as shown in Figure 7(b), in which the error given by this metric reaches maximum where the same pair of parameters produces minimal error when measured by total execution time. The extortion is also captured by MPI histogram and node-to-node timing, as shown in Figures 7(c) and 7(d). Both of these metrics give maximum error value where total execution time reports minimal error. While the error given by total execution time keeps constant throughout most of the parameter space, the errors given by the fine-grained metrics fluctuates, indicating a higher level of sensitivity in the parameter space considered. For example, on 768 processes, the error given by total execution time ranges from 0.003 to 0.004 with a small variance of $20 \%$. Moreover, the collective synchronization metric reveals correct performance trade-off between switch bandwidth and latency: an increase in switch latency can be compensated by an increase in switch bandwidth. As shown by the dark blue strip in Figure 7(b), the error given by the collective synchronization metric converges steadily toward a line that is parallel to the diagonal.

In Figure 8, we show the error when sweeping the BTE bandwidth and latency using coMD. In Figure 8(a), we observe that the error given by total execution time converges at around $3 \mathrm{~GB} / \mathrm{s}$. The same convergence trend is captured by the per-node timing metric in Figure 8(b), but with larger slope around the minimum. The improved contrast indicates better fidelity, but the per-node metric is still highly correlated to the total execution time because the timing break down is coarse-grained. The error given by the collective synchronization metric also converges at the same bandwidth as shown in Figure 8(c). Additionally, this metric exhibits sensitivity for BTE latency by reporting smaller error for larger latency. In Figure 8(d), we show the error given by the 

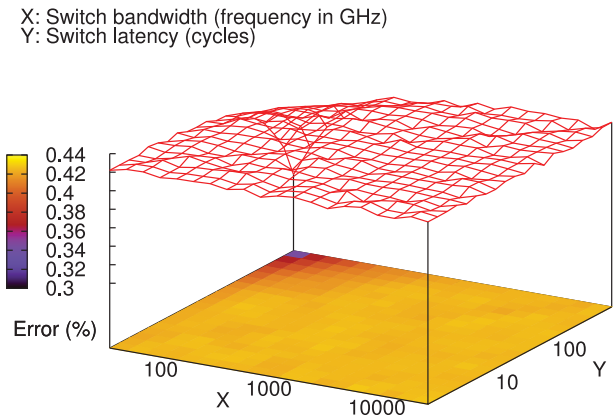

(a) Total execution time 768 processes

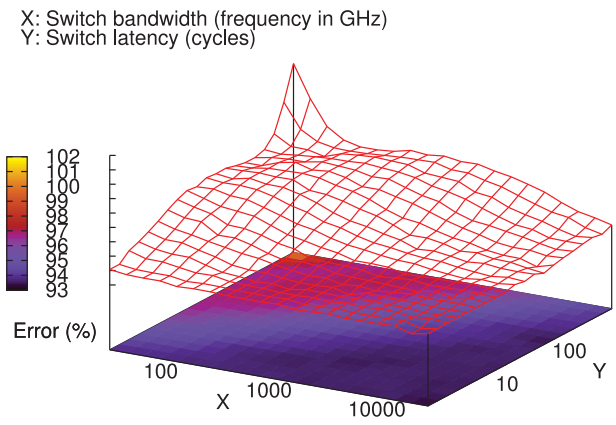

(c) MPI histogram 1,536 processes

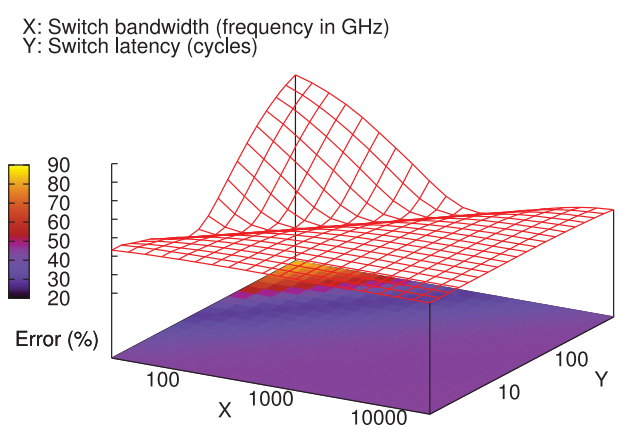

(b) Collective synchronization 768 processes

X: Switch bandwidth (frequency in $\mathrm{GHz}$ ) Y: Switch latency (cycles)

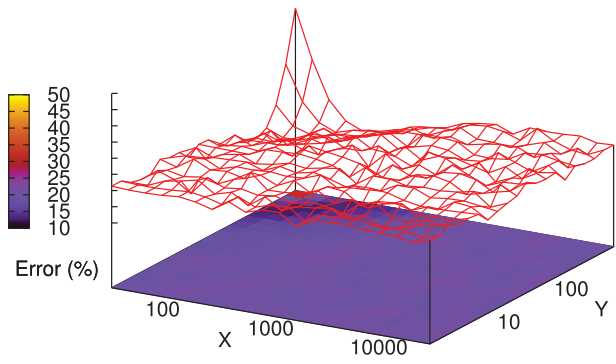

(d) Node-to-node timing 3,072 processes

Fig. 7. Switch bandwidth-latency sweeping for coMD.

node-to-node timing metric for 3,072 processes. We observe that the metric correctly divided the execution into two groups, which is indicated by a cliff on the error plane at around $7 \mathrm{~GB} / \mathrm{s}$. The undulation of the error plane in Figure $8(\mathrm{~d})$ is more frequent that that in Figure 8(a). This is an indication that node-to-node timing is more sensitive to the subtle changes of execution. However, this high sensitivity also makes it easy to be affected by noise: the error does not show a clear convergence trend to the right of $7 \mathrm{~GB} / \mathrm{s}$.

In Figure 9, we show the error when sweeping the FMA bandwidth and latency using coMD. In Figure 9(a), we observe the error given by the total execution time fluctuates within a small range $(0.41 \%-0.43 \%)$, which means that the FMA path has little contribution to the total execution time. The error given by total execution for larger scales exhibit the same pattern. Note that the FMA path generally utilizes UDP as the underlying communication protocol for small messages; it is especially susceptible to noise. In Figure 9(b), we show the error given by node-to-node timing for 3,072 processes. The error plane fluctuates as in the case of total execution time due to noise. In Figure 9(c), the MPI histogram metric diminishes the noise and produces error that consistently prefers smaller FMA latency. The fact that the FMA path is intended for small messages is consistent with the error changes in the $y$-axis: latency plays a more important role than bandwidth for occasional short communication among nodes. In Figure 9(d), the error given by the collective synchronization metric supports this 


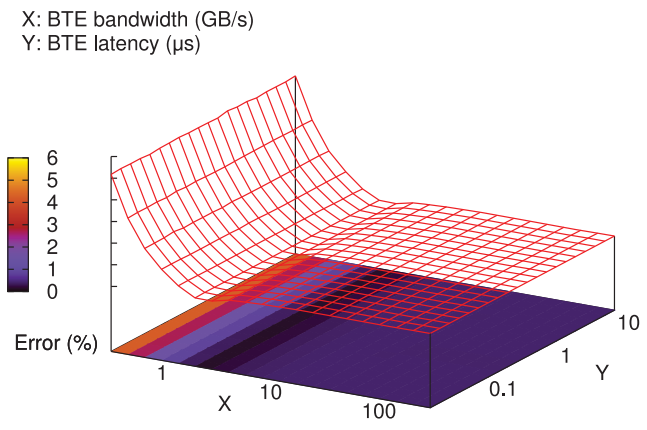

(a) Total execution time for 768 processes $\mathrm{X}$ : BTE bandwidth $(\mathrm{GB} / \mathrm{s})$
$\mathrm{Y}$ : BTE latency $(\mu \mathrm{s})$

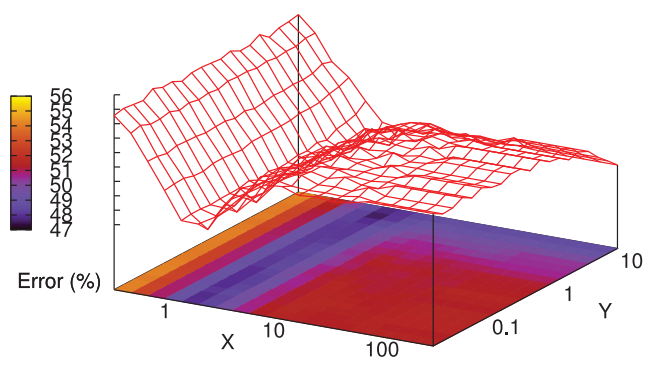

(c) Collective synchronization for 1,536 processes

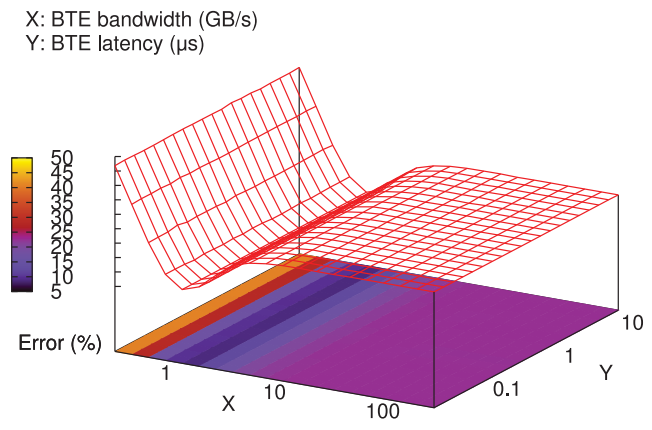

(b) Per node for 768 processes

$\mathrm{X}$ : BTE bandwidth $(\mathrm{GB} / \mathrm{s})$ Y: BTE latency $(\mu \mathrm{s})$

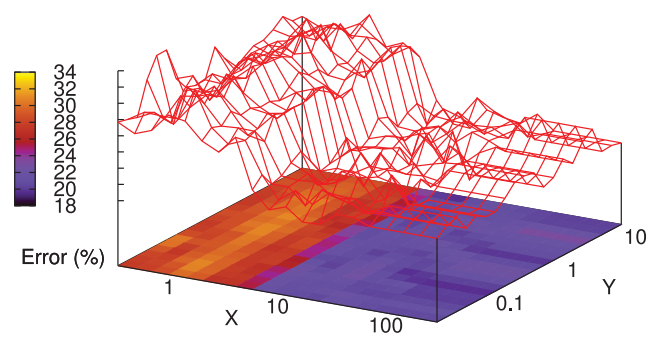

(d) Node-to-node timing for 3,072 processes

Fig. 8. BTE bandwidth-latency sweeping for coMD.

observation, and it further reveals an optimal latency of $7 \mu \mathrm{s}$ that minimizes the error of simulation against the real execution.

To conclude, our fine-grained evaluation metrics accurately capture the characteristics of parallel execution. Manual analysis of the metrics reveals additional insight into the disparity between the simulation and real execution. More importantly, when the errors are quantized, the metrics serve as reliable criteria for automatically identifying the optimal hardware model. In parameter spaces where traditional total execution time cannot be applied effectively, they provide additional details that are valuable for simulation tuning. In the presence of lite noise, the statistical features stabilize the metrics. However, as the raw difference between total execution time increases, any fine-grained details are likely to be dwarfed by the overall timing error. The error values given by the fine-grained metrics may be no longer reliable, but we can resort to manual inspection.

\section{RELATED WORK}

Although literature on MPI simulation contains rigorous discussion on the evaluation methods applied to establish the reliability of the results, to the best of our knowledge, there is no dedicated work addressing fine-grained simulation validation. For a simulation framework concerned with the overall performance prediction, evaluating accuracy with total execution time is the most intuitive choice since it is directly linked to performance. It is also the most pervasive evaluation metric adopted by a large 
X: FMA bandwidth $(\mathrm{GB} / \mathrm{s})$
Y: FMA latency $(\mu \mathrm{s})$

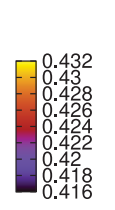

Error (\%)

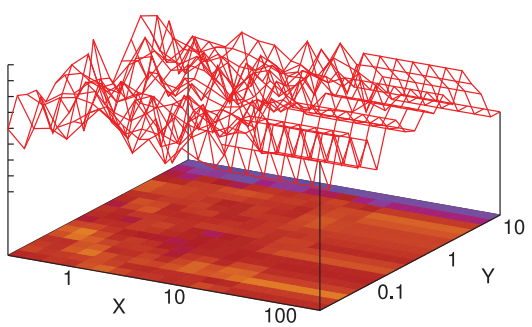

(a) Total execution time for 768 processes

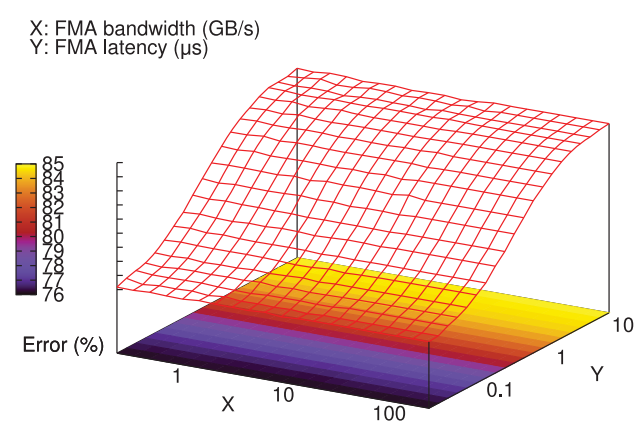

(c) MPI histogram for 768 processes
X: FMA bandwidth $(\mathrm{GB} / \mathrm{s})$
Y: FMA latency $(\mu \mathrm{s})$

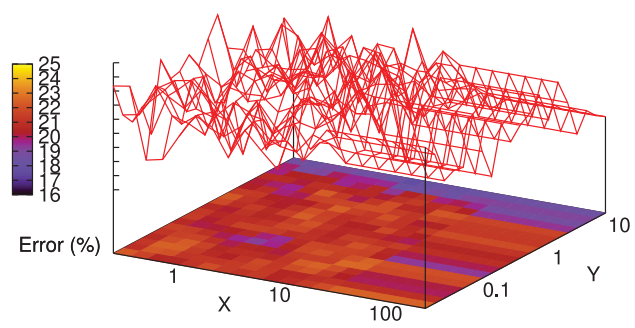

(b) Node-to-node timing for 3,072 processes X: FMA bandwidth $(\mathrm{GB} / \mathrm{s})$
Y: FMA latency $(\mu \mathrm{s})$

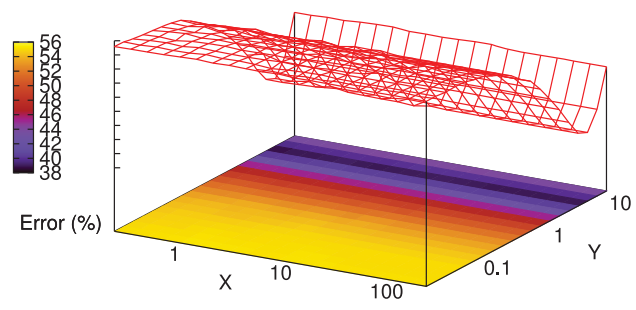

(d) Collective synchronization for 1,536 processes

Fig. 9. FMA bandwidth/latency sweeping for coMD.

number of researchers to validate their simulation framework [Desprez et al. 2011; Zhai et al. 2010; Núñez et al. 2010; Hoefler et al. 2010; Clauss et al. 2011; Penoff et al. 2009; Hammond et al. 2009; Zheng et al. 2004].

\subsection{Fine-Grained Metrics}

Underwood et al. [2007] use network latency and network bandwidth to validate their network models against Red Storm. Denzel et al. [2010] use network throughput besides execution time to validate the interconnect design. In the study by Velho and Legrand [2009], network throughput and communication time is used to validate the flow-based network model. The preceding network related metrics targeted the verification of specific hardware components, and are not sufficient to serve as an all-around validation metric for a system simulator as a whole. PSINS [Tikir et al. 2009] has a built-in statistical module that profiles the simulated application. It not only compares communication time but also provides a breakdown comparison between the time spent in each MPI routine. They show that the simulated results match the measured results at a finer granularity. Their approach bears a close resemblance to ours in that finegrained statistics are used. However, they use statistical data on communication time only, while in our proposed approach we take into account other aspects of a parallel execution such as load balancing, traffic, and synchronization. More importantly, in their validation methodology the time breakdown is compared qualitatively. Instead, we developed a quantitative error measure for all statistical data. Another relevant 
approach is presented in the validation of MicroGrid [Song et al. 2000], where the authors monitor an internal variable value through an identical performance tool. ${ }^{6}$ The periodical changes of the value in both real and simulated environment are sampled and compared. The fitness between the two variable data tells the similarity of the program's internal execution status. This method shares the same insight as the use of fine-grained statistics in that they both capture the internal characteristics of the execution. Comparing with our approach, the drawback of monitoring an internal variable is that the necessary variable choice is program dependent and the monitoring process is intrusive.

\subsection{Tracing and Profiling}

Recent progress on communication tracing opens up opportunities for performance studies based on scrutiny of event traces. Wu and Mueller [2011] devise an approach for topology extrapolation of communication traces. They synthetically generate the application trace for large numbers of nodes from a set of smaller traces, which enables the study of communication behavior at arbitrary scale without actual execution at that scale. Preissl et al. [2008] present an algorithm to detect and extract MPI communication patterns from traces. They detect repeating communication behavior to help identify possible performance bottlenecks. Weber et al. [2012] propose a trace comparison algorithm that aligns the traces of similarly behaving applications. They adopt a sequence alignment algorithm to align and compare the traces event-wise. The preceding approaches all highlight execution details and facilitate manual inspection of trace files. Besides these, traditional profilers like IPM [Wright et al. 2009] and mpiP [Vetter and McCracken 2001] have been extensively used for optimization of parallel application. Vampir [Nagel et al. 1996], Scalasca [Geimer et al. 2010], and TAU [Shende and Malony 2006] are some of the well known comprehensive tracing tools. Our toolset bears a resemblance to the preceding approaches in that it captures execution details and allows manual inspection of runtime characteristics. The key difference is that our toolset prepares statistics pertaining to hardware parameters in matrix format, which facilitates quantitative comparison of traces from multiple dimensions. This is essential in simulation validation tasks and makes a step toward automating the simulation tuning process.

\subsection{Error Measure}

The most common choice is the percentage error or relative error. It is defined as

$$
\operatorname{Err}=\frac{X-R}{R}
$$

where $R$ is the reference value and $X$ is the experiment value. When validating the simulation result, the predicted total execution time $X$ is compared against the actual execution time $R$. The major drawback is that the relative error is not symmetric. To overcome the biases introduced by relative error metric, logarithmic error was proposed in the accuracy study of SimGrid [Velho and Legrand 2009]. It is defined as

$$
\log E r r=|\log X-\log R| .
$$

The error function now is symmetrical; this can be seen from

$$
|\log X-\log R|=|\log R-\log X| .
$$

\footnotetext{
${ }^{6}$ The tool that tracks the values of certain types of program variables over the execution of the program. Refer to the work of Song et al. [2000] for more details.
} 
Not only is it symmetrical, one can now perform aggregation operations like the maximum, the mean, and the variance. To interpret logarithmic error as percentage, additional conversion is needed to take the value out of log-space:

$$
E r r=e^{\log E r r}-1 .
$$

SMPI [Clauss et al. 2011] is another platform that applies this more robust metric in their evaluation process. In addition to symmetry, an important property of logarithmic error is that it gives the same error regardless of the units (communication time or bandwidth) used to measure the network performance. Since the proposed metrics only evaluate system performance in time, we prefer percentage differences for simplicity.

\subsection{Benchmark}

Benchmark packages fall into three levels with increasing complexity. Synthetic benchmarks often consist of a set of very short tests that stress a particular component. SKaMPI [Reussner et al. 1998] is such a package aiming at providing precise communication timing for MPI routines. Mini-applications are self-contained programs that capture the essential or frequently invoked computation and communication behavior of a full application. They often stem from actual scientific applications like fluid dynamics or Poisson solvers, but can run without lengthy setup and full input data. Since mini-applications behave much like their real-world counterparts, they are a popular choice for simulation validation. A few of these mini-applications have become standardized test programs such as the NAS Parallel Benchmarks (NPBs) and Sweep3D. The most sophisticated benchmark is a full-blown application, which provides the most accurate rating of the simulation. However, running full applications is both time and resource consuming, especially in a single node simulation environment where the execution speed is much slower than a physical cluster. While most works on simulation framework adopt only one of the preceding benchmarks, MicroGrid [Song et al. 2000] encompasses the most comprehensive validation process using all of the three. MicroGrid's approach begins with individual tests of a single resource model with synthetic benchmarks to show that the tested components work correctly in isolation, then NPB is used to test the combined accuracy of the system, and finally a full application (Cactus PDE solver) is employed to further validate the utility and fidelity. While this validation process yields a thorough evaluation report, it is often not practical to employ such complex tests. In single node simulation scenario, it is often not feasible to simulate full application online. In our approach, we gain a considerable level of knowledge about executions through the use of our fine-grained metrics, so we can adopt lightweight mini-applications to speed up simulation without loss of details.

\section{CONCLUSION AND FUTURE WORK}

In this article, we presented the first fine-grained accuracy evaluation approach for large-scale parallel application simulations. The proposed metrics capture the signature of parallel execution traces by aggregating timing and traffic information into matrices. In our experimental study, we illustrated the parameter insensitivity problem that occurs during simulation in high-dimensional parameter space, and showed that the proposed statistical metrics provide improved fidelity and extra coverage when compared to the typical approach of using total execution time. We designed and implemented a toolset for both hardware model and software model validation, which works with SST/macro and its DUMPI library. The toolset can also be applied to other parallel application simulation frameworks.

Based on the results of automated error analysis in Section 4.2, future work will focus on building an automatic simulation tuning framework that finds optimal simulation parameters with little or no human intervention. This framework would heuristically 
search through the high-dimensional simulation parameter space, including categorical and discrete parameters, and favor those that produce the most similar traces. An automated tuning framework will enable quantitative exploration of design trade-offs and efficient porting of HPC applications in the hardware/software codesign process. It can be combined with the work on uncertainty assessment [Wilke et al. 2013] to provide reliable evaluation of the accuracy of simulation framework.

The proposed approach extends naturally to the evaluation of other computational resources, such as I/O traffic and energy consumption, given that the resource activities are logged by the tracing tools. For example, cycle accurate simulation framework SST [Rodrigues et al. 2011] models chip power consumption. When used in conjunction with our approach, energy signature of the execution could be extracted and analyzed. It is also possible to extend this approach to other programming paradigms such as OpenMP and MapReduce. OpenMP is similar to MPI in that it allows for explicit synchronization among threads. The barrier clause in OpenMP effectively divides the program into synchronization phases similar to that of a MPI program. Aggregated performance analysis and comparison is possible if the execution of OpenMP programs is traced [Huck et al. 2014]. The node-to-node communication and collective synchronization metrics can also be applied to the measure the shuffle stage of a MapReduce algorithm.

\section{REFERENCES}

C. Albing, N. Troullier, S. Whalen, R. Olson, J. Glenski, H. Pritchard, and H. Mills. 2011. Scalable node allocation for improved performance in regular and anisotropic 3D torus supercomputers. In Recent Advances in the Message Passing Interface. Springer, 61-70.

P. N. Clauss, M. Stillwell, S. Genaud, F. Suter, H. Casanova, and M. Quinson. 2011. Single node on-line simulation of MPI applications with SMPI. In 2011 IEEE International Parallel \& Distributed Processing Symposium (IPDPS). IEEE, 664-675.

W. E. Denzel, J. Li, P. Walker, and Y. Jin. 2010. A framework for end-to-end simulation of high-performance computing systems. Simulation 86, 5-6 (2010), 331-350.

F. Desprez, G. S. Markomanolis, M. Quinson, and F. Suter. 2011. Assessing the performance of MPI applications through time-independent trace replay. In Proceedings of the 2011 40th International Conference on Parallel Processing Workshops (ICPPW). IEEE, 467-476.

M. Geimer, F. Wolf, B. J. N. Wylie, E. Ábrahám, D. Becker, and B. Mohr. 2010. The Scalasca performance toolset architecture. Concurrency and Computation: Practice and Experience 22, 6 (2010), 702-719.

S. D. Hammond, G. R. Mudalige, J. A. Smith, S. A. Jarvis, J. A. Herdman, and A. Vadgama. 2009. WARPP: A toolkit for simulating high-performance parallel scientific codes. In Proceedings of the 2nd International Conference on Simulation Tools and Techniques. ICST (Institute for Computer Sciences, SocialInformatics and Telecommunications Engineering), 19.

T. Hoefler, T. Schneider, and A. Lumsdaine. 2010. LogGOPSim-simulating large-scale applications in the LogGOPS model. In Proceedings of the 19th ACM International Symposium on High Performance Distributed Computing (HPDC), Vol. 10. 597-604.

K. A. Huck, A. D. Malony, S. Shende, and D. W. Jacobsen. 2014. Integrated measurement for cross-platform OpenMP performance analysis. In Using and Improving OpenMP for Devices, Tasks, and More. Springer, $146-160$.

C. L. Janssen, H. Adalsteinsson, S. Cranford, J. P. Kenny, A. Pinar, D. A. Evensky, and J. Mayo. 2010. A simulator for large-scale parallel computer architectures. International Journal of Distributed Systems and Technologies 1, 2 (2010), 57-73.

C. Janssen, D. Quinlan, and J. Shalf. 2011. Architectural simulation for exascale hardware/software co-design.

S. Kamil, L. Oliker, A. Pinar, and J. Shalf. 2010. Communication requirements and interconnect optimization for high-end scientific applications. IEEE Transactions on Parallel and Distributed Systems 21, 2 (2010), 188-202.

W. E. Nagel, A. Arnold, M. Weber, H. C. Hoppe, and K. Solchenbach. 1996. VAMPIR: Visualization and Analysis of MPI Resources. Citeseer. 
A. Núñez, J. Fernández, J. D. Garcia, F. Garcia, and J. Carretero. 2010. New techniques for simulating high performance MPI applications on large storage networks. The Journal of Supercomputing 51, 1 (2010), 40-57.

A. J. Peña, R. G. C. Carvalho, J. Dinan, P. Balaji, R. Thakur, and W. Gropp. 2013. Analysis of topologydependent MPI performance on Gemini networks. In Proceedings of the 20th European MPI Users' Group Meeting. ACM, 61-66.

B. Penoff, A. Wagner, M. Tuxen, and I. Rungeler. 2009. MPI-NetSim: A network simulation module for MPI. In Proceedings of the 2009 15th International Conference on Parallel and Distributed Systems (ICPADS). IEEE, 464-471.

R. Preissl, T. Kockerbauer, M. Schulz, D. Kranzlmuller, B. Supinski, and D. J. Quinlan. 2008. Detecting patterns in MPI communication traces. In Proceedings of the 37th International Conference on Parallel Processing (ICPP'08). IEEE, 230-237.

R. Reussner, P. Sanders, L. Prechelt, and M. Müller. 1998. SKaMPI: A detailed, accurate MPI benchmark. Recent Advances in Parallel Virtual Machine and Message Passing Interface (1998), 52-59.

A. F. Rodrigues, K. S. Hemmert, B. W. Barrett, C. Kersey, R. Oldfield, M. Weston, R. Risen, J. Cook, P. Rosenfeld, E. CooperBalls, and others. 2011. The structural simulation toolkit. ACM SIGMETRICS Performance Evaluation Review 38, 4 (2011), 37-42.

J. Shalf, D. Quinlan, and C. Janssen. 2011. Rethinking hardware-software codesign for exascale systems. Computer 44, 11 (2011), 22-30.

S. S. Shende and A. D. Malony. 2006. The TAU parallel performance system. International Journal of High Performance Computing Applications 20, 2 (2006), 287-311.

H. J. Song, X. Liu, D. Jakobsen, R. Bhagwan, X. Zhang, K. Taura, and A. Chien. 2000. The microgrid: A scientific tool for modeling computational grids. In Proceedings of the ACM/IEEE 2000 Conference on Supercomputing. IEEE, 53-53.

M. Sottile, A. Dakshinamurthy, G. Hendry, and D. Dechev. 2013. Automatic extraction of software skeletons for benchmarking large-scale parallel applications. In ACM SIGSIM Conference on Principles of Advanced Discrete Simulation (PADS).

C. D. Sudheer and A. Srinivasan. 2012. Optimization of the hop-byte metric for effective topology aware mapping. In Proceedings of the 2012 19th International Conference on High Performance Computing (HiPC). IEEE, 1-9.

R. Susukita, H. Ando, M. Aoyagi, H. Honda, Y. Inadomi, K. Inoue, S. Ishizuki, Y. Kimura, H. Komatsu, M. Kurokawa, and others. 2008. Performance prediction of large-scale parallell system and application using macro-level simulation. In Proceedings of the 2008 ACM/IEEE Conference on Supercomputing. IEEE, 20.

M. Tikir, M. Laurenzano, L. Carrington, and A. Snavely. 2009. PSINS: An open source event tracer and execution simulator for MPI applications. Euro-Par 2009 Parallel Processing, 135-148.

K. D. Underwood, M. Levenhagen, and A. Rodrigues. 2007. Simulating red storm: Challenges and successes in building a system simulation. In IEEE International Parallel and Distributed Processing Symposium (IPDPS 2007). IEEE, 1-10.

P. Velho and A. Legrand. 2009. Accuracy study and improvement of network simulation in the SimGrid framework. In Proceedings of the 2nd International Conference on Simulation Tools and Techniques. ICST (Institute for Computer Sciences, Social-Informatics and Telecommunications Engineering), 13.

J. S. Vetter and M. O. McCracken. 2001. Statistical scalability analysis of communication operations in distributed applications. In ACM SIGPLAN Notices, Vol. 36. ACM, 123-132.

M. Weber, R. Brendel, and H. Brunst. 2012. Trace file comparison with a hierarchical sequence alignment algorithm. In Proceedings of the 2012 IEEE 10th International Symposium on Parallel and Distributed Processing with Applications (ISPA). IEEE, 247-254.

J. J. Wilke, K. Sargsyan, J. P. Kenny, B. Debusschere, H. N. Najm, and G. Hendry. 2013. Validation and uncertainty assessment of extreme-scale HPC simulation through Bayesian inference. In Euro-Par 2013 Parallel Processing. Springer, 41-52.

N. J. Wright, W. Pfeiffer, and A. Snavely. 2009. Characterizing parallel scaling of scientific applications using IPM. In Proceedings of the 10th LCI International Conference on High-Performance Clustered Computing. 10-12.

X. Wu and F. Mueller. 2011. ScalaExtrap: Trace-based communication extrapolation for SPMD programs. In Proceedings of the 16th ACM Symposium on Principles and Practice of Parallel Programming. ACM, 113-122.

J. Zhai, W. Chen, and W. Zheng. 2010. Phantom: Predicting performance of parallel applications on large-scale parallel machines using a single node. In ACM Sigplan Notices, Vol. 45. ACM, 305-314. 
D. Zhang, G. Hendry, and D. Dechev. 2014. Tools for enabling automatic validation of large-scale parallel application simulations. In 2014 IEEE International Conference on Software Maintenance and Evolution (ICSME). IEEE, 601-604.

G. Zheng, G. Kakulapati, and L. V. Kalé. 2004. Bigsim: A parallel simulator for performance prediction of extremely large parallel machines. In Proceedings of the 18th International Parallel and Distributed Processing Symposium, 2004. IEEE, 78.

Received November 2014; revised May 2015; accepted July 2015 